\title{
Cytogenetic harvesting of commonly used tumor cell lines
}

\author{
Roderick A F MacLeod, Maren Kaufmann \& Hans G Drexler \\ DSMZ—German Collection of Microorganisms and Cell Cultures, Braunschweig, Germany. Correspondence should be addressed to R.A.F.M. (rml@dsmz.de). \\ Published online 8 March 2007; doi:10.1038/nprot.2007.29

\begin{abstract}
Tumor cell lines are widely used both as disease models and, increasingly, as genomic resources for the ascertainment of new cancer genes. Cytogenetic analysis remains a major route to uncovering the cancer genome. However, cancer cell lines vary inexplicably in their harvesting preferences, which must, therefore, be determined by trial and error. This article describes harvesting protocols optimized empirically for 550 commonly used, mainly human, cancer cell lines together with evidence-based procedures to assist in determining conditions for unlisted cell lines and subsidiary protocols for cytogenetic analysis using G-banding and fluorescence in situ hybridization.
\end{abstract}

\section{INTRODUCTION}

Need for tumor cell line cytogenetic harvesting methods Continuous cell lines allow cumulative, verifiable investigations that are impracticable with primary material alone. Among these, cytogenetic analysis is core, notably in tumor and neonatal pathology. The provision of detailed, comprehensive mapping and sequencing data by the Human Genome Project, together with bacterial artificial chromosome (BAC) and fosmid clones, has enabled cytogenetic analysis using fluorescence in situ hybridization (FISH) to bridge the gap between cells and molecules, enabling identification of many cancer genes targeted by recurrent chromosome rearrangements, as seen in a wide variety of tumors.

Hence, well-characterized neoplastic cell lines provide ideal ground for mining new cancer genes using classical cytogenetics and FISH - both routinely performed on metaphase chromosome preparations. However, such efforts may be frustrated by the unexpected difficulty of obtaining adequate metaphase chromosome preparations from many tumor cell lines using standardized procedures, which is exacerbated by the complexity of chromosome changes therein. Although standard cytogenetic harvesting methods may suit the narrow range of primary diagnostic material normally encountered (notably peripheral blood lymphocytes, amniocytes and skin biopsies), other types of primary cell or tumor are poorly catered for. We have determined optimized conditions for harvesting metaphase chromosome preparations from cell lines held at a large general cell repository.

Some 1,500 human hematopoietic cell lines have been established $^{1,2}$, which would extrapolate to a grand total approaching 10,000 for all tumor types. Few of these have been cytogenetically analyzed in detail. This is regrettable, as cytogenetic data inform both authenticity and likely derivation. In addition, chromosome rearrangements often indicate genes altered in cancer: Cancer Genome Project data show that 273/360 known cancer genes (76\%) participate in chromosome rearrangements (http:// www.sanger.ac.uk/genetics/CGP/Census/chromosome.shtml) and are thus potentially ascertainable using cytogenetic methods. Unsurprisingly, cytogenetic data afford a uniquely informative global perspective on individual cancer cells and, by reference to previously published karyotypes, assist in detecting cross-contamination-a chronic unresolved problem ${ }^{3}$.
Given their potentially unlimited supply, it might be reasonably supposed that continuous cell lines pose fewer significant technical challenges for cytogenetic harvesting than primary cultures. Such is not the case, alas! In contrast to primary tissues, tumor cells and derived cell lines display enormous variations in origins and states of development. Cell lines are often extremely sensitive to the culture microenvironment and grow poorly at low cell densities. It is now recognized that mitotic scarcity or inadequate chromosome morphology epitomizes certain tumor types ${ }^{4,5}$. Tellingly, overexpression of a key leukemia oncogene myeloid-lymphoid or mixedlineage leukemia (MLL) has been recently shown to control chromatin de-condensation ${ }^{6}$, offering a possible clue to the poor chromosome morphologies often encountered therein.

Although there is some correlation between cell or tumor type and choice of harvesting procedure, it falls short of a rigorous guiding principle. Accordingly, we describe protocols optimized in our labs for harvesting each of 550 commonly used cancer cell lines, accompanied by evidence-based procedures for determining optimal harvesting conditions for unlisted cell lines. In addition, modular protocols are described for related cytogenetic methods, namely, trypsin G-banding, non-isotopic probe labeling using nick-translation (NT) and FISH.

\section{Cytogenetic methodologies}

It first became routine in the 1970s to distinguish each of the 24 different human chromosomes when methods for recognizing substructural bands were reported, notably Q(uinacrine)-banding ${ }^{7}$ and trypsin G-banding ${ }^{8}$, which enabled identification of the 'Philadelphia chromosome' ( $\mathrm{Ph}$ ) marker in chronic myeloid leukemia $(\mathrm{CML})$ and its formation through a reciprocal translocation, $t(9 ; 22)(q 34 ; q 11)^{9}$. This observation directly led to cancerogenesis being attributed to specific gene alterations. The advent of FISH during the late 1980s (refs. 10, 11) enabled genomic interrogation using region-specific probes that were labeled non-isotopically by NT and visualized by fluorescence microscopy. This in turn led to chromosome- and, subsequently, gene-specific probes becoming commercially available. Subsequently, spectral karyotyping (SKY) and $\mathrm{m}$ (ultiplex)-FISH, rendered visible global cytogenetic alterations in cancer cells ${ }^{12}$. In addition, comparative genomic hybridization (CGH), which uses mitotic metaphase chromosomes as a 


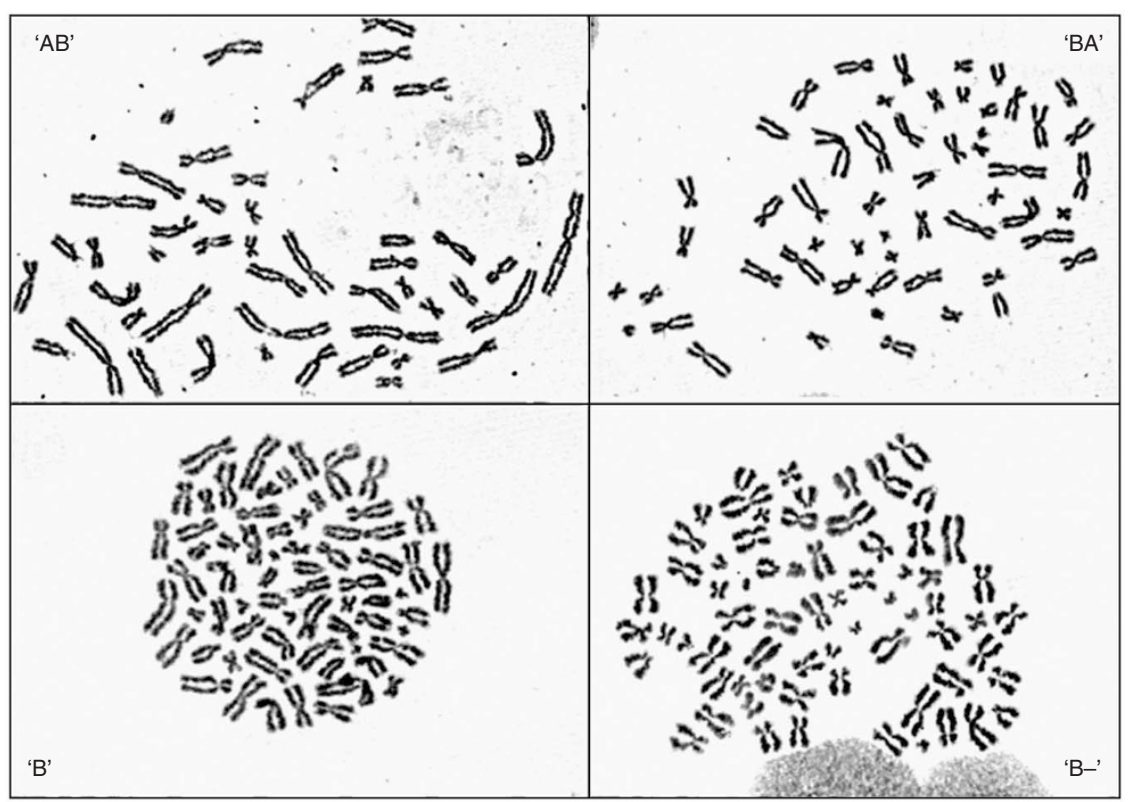

Figure 1 | Morphology. Images depict human metaphase chromosomes from cancer cells assessed at four critical morphological quality levels as used in Supplementary Table 1: AB, BA, B and B-. Human cancer chromosomes rarely exceed $A B$ in morphology. The metaphase classed $B$ - would be unlikely to yield adequate G-banding or FISH preparations. (Note that all metaphases display 'spreading' assessed as A.)

platform, allowed the detection of elusive genomic deletions and amplifications, inspiring more sensitive a(rray)-CGH chip-based methods.

Most recently, mapped and sequenced bacterial/P1 artificial chromosome (BAC/PAC) clones made available as a result of the Human Genome Project have allowed suitably equipped investigators to dissect chromosome rearrangements at the level of single genes and smaller. In this protocol, we describe basic cytogenetic procedures that have been adapted in our laboratory for use with cell cultures. For those planning de novo cytogenetic analysis of tumor cell lines, it is convenient to split the task into the following steps: harvesting, G-banding, NT and FISH (see Step 22).

\section{Notes about the procedure}

Harvesting and slide preparation are crucial for subsequent success with both G-banding and FISH. Indeed, hypotonic treatments that consistently yield good preparation with one cell line may be unsuitable for its congener, hampering use of standard harvesting protocols. It is, therefore, necessary to determine optimized harvesting procedures for each cell line by trial and error using parallel cell aliquots exposed to a range of hypotonic conditions by varying buffers, durations and, if necessary, the temperatures of each treatment. Cytogenetic harvesting is extremely sensitive to biological variability and must often be repeated until satisfactory results are achieved.
Fixation, in contrast, permits standardization. Fixed cells may be stored for a few years at $-20{ }^{\circ} \mathrm{C}$ until required, whether for Gbanding or FISH. Immediately before slide making, cryostored cell suspensions should be washed in fresh fixative. Slide making is performed by dropping a suspension onto ice-cold, 'squeaky-clean' slides held at a slight incline (approximately $15^{\circ}$ ) on top of a prefrozen $\left(-20{ }^{\circ} \mathrm{C}\right)$ freezer cold-block. Two drops aimed immediately under the frosted zone and at the lower middle, respectively, produce figure-of-eight spreading patterns that facilitate both G-banding and FISH (Figs. 1 and 2). Once made, slides may be stored for a few years at $-80^{\circ} \mathrm{C}$ for FISH, or aged overnight at $60{ }^{\circ} \mathrm{C}$ for G-banding.

G-banding (the most popular banding method) involves trypsin pre-treatment, which depletes chromatin of certain proteins to produce strong lateral bands after staining with Giemsa. Analysis of chromosomes harvested using this protocol should typically reveal some 300 bands per haploid genome ${ }^{13}$. With G-banding alone, it is possible to detect, if not fully characterize, most chromosome rearrangements present in cell lines carrying up to approximately half a dozen separate changes; i.e., it is sufficient for confirming cell line identity. The utility of Gbanding in detecting cell line cross-contamination is illustrated in Figure 3, which shows CCRF-CEM derived from pediatric T-acute lymphoblastic leukemia (T-ALL) and its tetraploid derivate COLE, mistakenly published as a Hodgkin lymphoma cell line $e^{3,14}$.

Chromosome painting aptly describes the FISH application, which uses probe libraries comprising anonymous DNA sequences

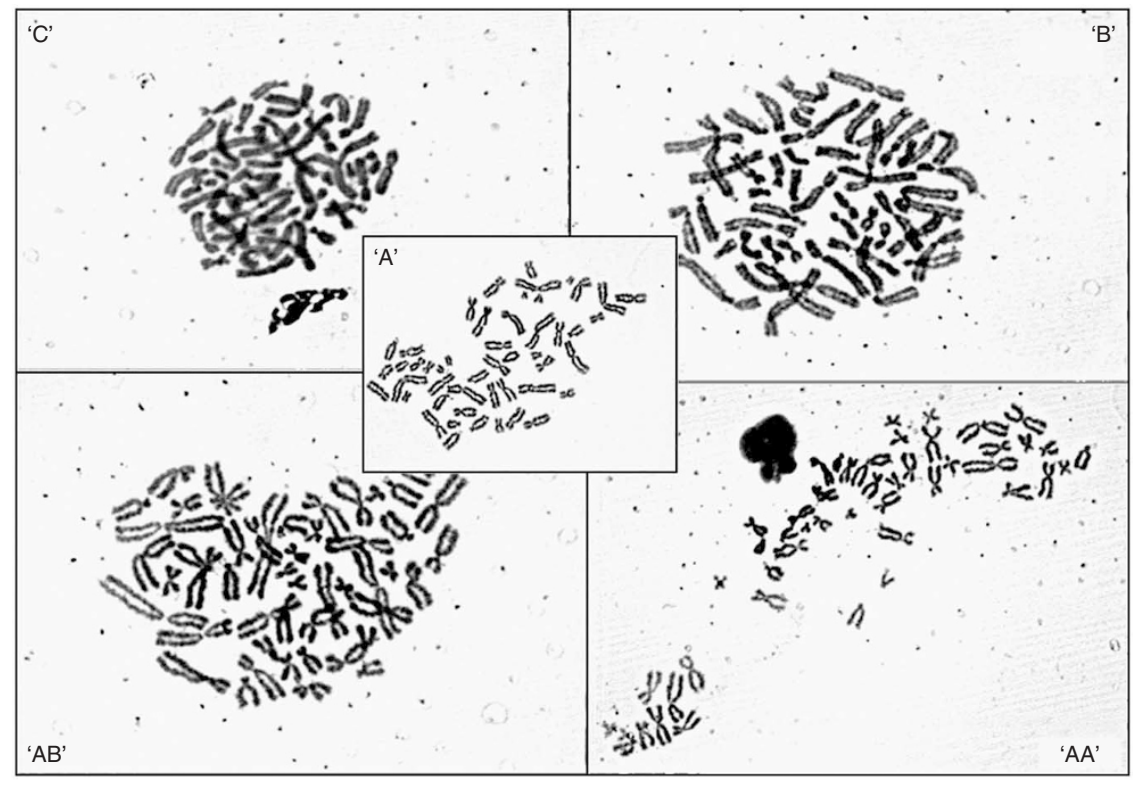

Figure 2 | Spreading. Images show critical reference levels for chromosome spreading. For G-banding one should aim for a minimum level of $A B$, whereas FISH experiments may be performed using metaphases classed as B. Optimal spreading, classed as A, is shown in the center for reference. 
Figure 3 | Authentication. (a) The G-banding karyogram depicts CCRF-CEM (DSM ACC 240). CCRF-CEM (T-cell leukemia) is karyotypically unstable, and early passages of this cell line are multiclonal. Thus CCRF-CEM has given rise to numerous subclones ${ }^{3,14},(\mathbf{b})$ one of which, COLE, is purportedly derived from Hodgkin's lymphoma. Note the tell-tale presence of der(8) (arrowheads) and $\operatorname{der}(9)$ (arrows) partners of $\mathrm{t}(8 ; 9)(\mathrm{p} 11 ; \mathrm{p} 24)$ in both karyograms. Reference karyotypes for cell lines listed in Supplementary Table 1 are given at http:// www.dsmz.de/human_and_animal_cell_lines/cell_line_index.php. Note the presence of two copies of der(9), but not der(8), marker chromosomes in COLE, deriving from a unique (at least among hematopoietic cell lines) $\mathrm{t}(8 ; 9)$ (p11;p24) rearrangement originally present in CCRF-CEM and testifying to their common identity. CCRF-CEM derivatives also carry additional copies of chromosome 20 (thin arrows). COLE is further distinguished from CCRF-CEM by unbalanced $\operatorname{der}(18) t(X ; 18)(p 11 ; q 11)$ rearrangements as well as by having evolved tetraploidy. CCRF-CEM was among the very first human leukemic cell lines to be established and has been widely distributed. Its unusual (i.e., among human leukemia cell lines) propensity to grow from clonal cell densities renders CCRF-CEM uniquely prone to 'bottlenecking selection' after over-dilution during extended culture. Perhaps this is why CCRF-CEM is the most prolific cross-contaminant of leukemic cell lines, each instance distinguished by $\mathrm{t}(8 ; 9)$ and trisomy 20 , together with a small number of secondary chromosome changes that conveniently serve as identifiers.

that hybridize to specific chromosomes or regions thereof. Painting probes may be used singly or, if contrastingly labeled, in combination (e.g., to confirm a translocation inferred by G-banding). Regardless of probe combination, it is usually necessary to counterstain the chromosomes. The standard counterstain, 4,6-diamidino2-phenylindole (DAPI), fluoresces deep blue, brightest at the centromeres, generating negative G-bands that can be digitally reversed.

Single-locus probes are produced by labeling plasmid or large insert clones, or obtained commercially: FISH using BAC clones covering

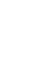

a

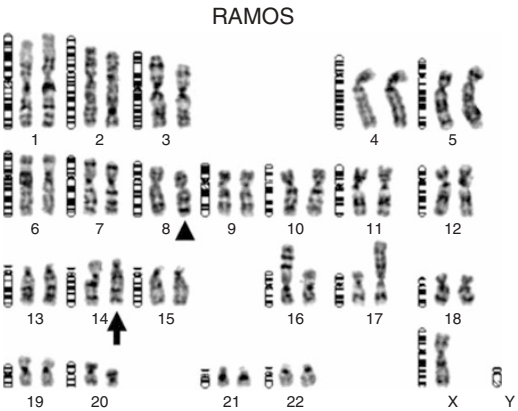

b

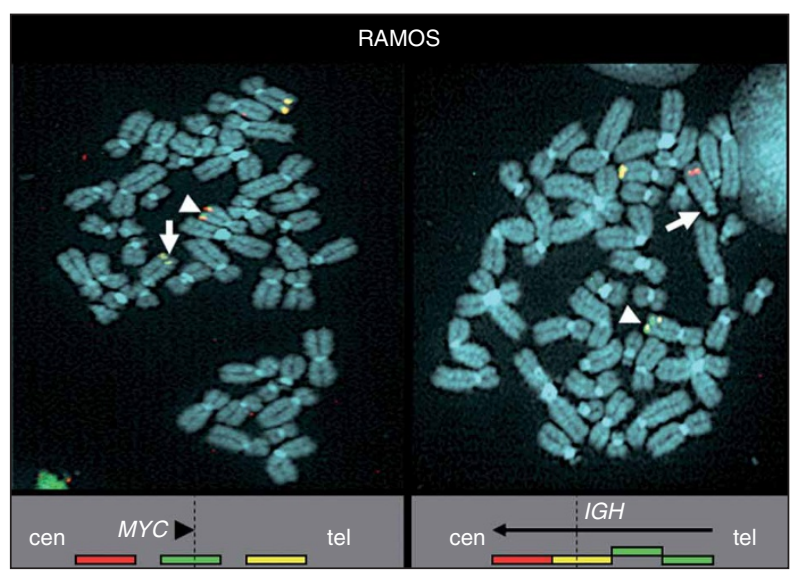

a CCRF-CEM

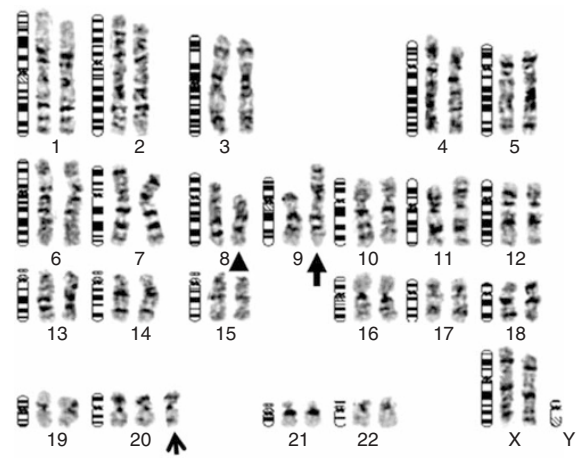

b

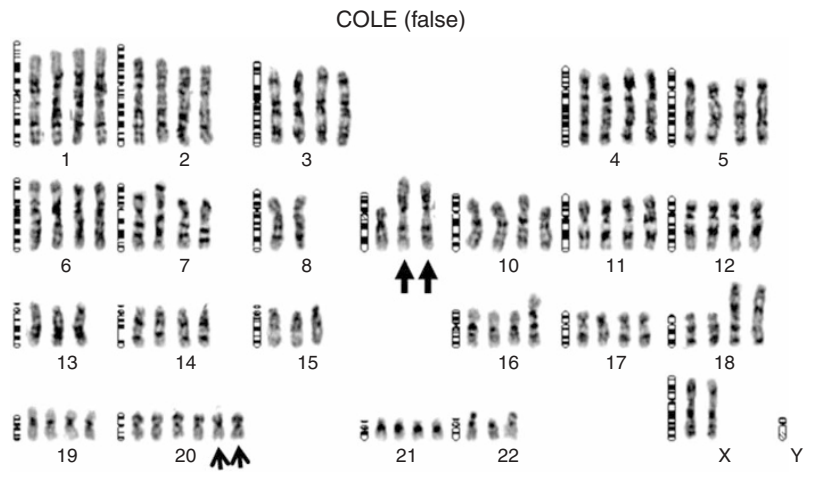

the myelocytomatosis viral oncogene homolog (MYC) and immunoglobulin heavy chain (IGH) loci at chromosomes 8q24 and 14q32 is shown in Figure 4. Such probes can be identified using genome browsers such as Ensembl (http://www.ensembl.org/index.html) or UCSC (http://genome.ucsc.edu/) and ordered from resource centers, e.g., BACPAC resources (http://bacpac.chori.org), RZPD—German Resource Center for Genome Research (http:// www.rzpd.de/) or the Sanger Institute (http://www.sanger.ac.uk/).

DNA preparation normally follows protocols given by the manufacturers of purification kits. We routinely use Big BAC DNA kits (Princeton Separations, Adelphia, NJ) labeled using standard non-isotopic NT protocols upon which the PROCEDURE

Figure 4 | Characterization. The (a) G-banding and (b) FISH images illustrate cytogenetic characterization of a Burkitt lymphoma cell line, RAMOS (DSM ACC 603). Arrowheads and arrows, respectively, indicate presence of the $\operatorname{der}(8)$ and $\operatorname{der}(14)$ partners of $t(8 ; 14)(q 24 ; q 32)$, which are typical of this tumor after both G-banding and FISH analysis using BAC clones covering the breakpoint regions of MYC (at 8q24) and IGH (at 14q32). In the G-banding karyogram, note additional rearrangements affecting chromosomes 14 (left), 16, 17 and 20. Below the FISH images are shown the approximate positions of the BAC clones, their labeling schemes, from cen(tromere) to tel(omere), respectively, and the breakpoints in RAMOS. The MYC breakpoint, near to MYC itself, is more typical of non-endemic Burkitt lymphomas, whereas endemic (African) cases often display more centromeric breakpoints. The IGH breakpoint is typical of $t(8 ; 14)$ and lies close to the powerful IGH E- $\mu$ transcriptional enhancer region. The BAC clones used for analysis of MYC at chromosome 8q24 (RP11 library clones obtained from the Sanger Centre, Cambridge, UK) were as follows (centromeric-telomeric): 828L6 (Spectrum Red), 440N18 (Spectrum Green), $195 \mathrm{G} 18$ (Cy3); and for analysis of IGH (obtained from Tim Poulsen, DAKO, Glostrup, Denmark; see ref. 17), 820M16 (Cy3), 11771 (Spectrum Red) and 448N5/151B17 (both Spectrum Green). 
is based. BAC probes often contain redundant sequences that crosshybridize to cause 'noise', which is partially suppressed by preincubating labeled probe DNA together with unlabeled Cot-1 DNA enriched for repetitive sequences. The critical step in FISH is the post-hybridization stringency wash performed either at low temperatures and including formamide, which lowers the stability of the DNA double helix, or at higher temperatures using low SSC (Sodium Chloride/Sodium Citrate) concentrations alone. Stringency washing allows the operator to balance probe signal intensity against background. For those starting with untested FISH probes, it is feasible to begin by using a less stringent wash that, if it yields unacceptable background levels, can be repeated at higher stringencies (i.e., at lower SSC concentrations).

The following procedure describes a flexible FISH protocol applicable to a wide variety of probes and usable by those intending to combine probes from different sources (i.e., for experiments with the highest informational value). FISH experiments with single commercial probes should normally be performed according to manufacturer's protocols, although these are unlikely to differ substantially from that presented here. Indirectly labeled probes (e.g., with digoxigenin or biotin) require additional detection steps, which may be plugged into the protocol described below.

\section{MATERIALS}

\section{REAGENTS}

- Cell culture(s) (maintained in logarithmic phase)

-5-Fluoro-2'-deoxyuridine $25 \mathrm{~g} \mathrm{~m} \mathrm{~m}^{-1}$ (FUDR; Sigma, Taufkirchen, Germany) (see REAGENT SETUP)

- $1-\beta$-D-ribofuranosyluracil $1 \mathrm{mg} \mathrm{ml}^{-1}$ (Urd; Sigma) (see REAGENT SETUP)

- Colcemid ( $N$-deacetyl- $N$-methylcolchicine; Invitrogen, Karlsruhe, Germany)

$4 \mu \mathrm{g} \mathrm{ml}^{-1}$ for $100 \times$ stock solution

- Thymidine (1-2-deoxy- $\beta$-D-ribofuranosyl-5-methyluracilthymide; Sigma)

(see REAGENT SETUP)

- Trypsin $0.5 \mathrm{~g} \mathrm{l}^{-1}$ (see REAGENT SETUP)

- Trypsin stock solution (see REAGENT SETUP)

- Fixative (see REAGENT SETUP)

- Spectrum Red and Spectrum Green dUTPs (Vysis, Bergisch Gladbach, Germany)

- Cy3 dUTP (GE Healthcare [formerly Amersham], Munich, Germany)

- Probe DNA (see REAGENT SETUP)

- $10 \times$ stop buffer: $0.3 \mathrm{M}$ EDTA (Invitrogen)

- $10 \mathrm{ml}$ carrier DNA from herring sperm (Serva, Heidelberg, Germany); use at

$10 \mathrm{mg} \mathrm{ml}^{-1}$; store aliquots frozen at $-20{ }^{\circ} \mathrm{C}$; this simultaneously acts as a carrier to enable subsequent visualization, a sacrificial barrier to adventitious enzymic degradation and a non-specific DNA cross-hybridization buffer to assist Cot-1 DNA

- Specific competitor DNA to use with probes containing repeated sequences:

$1 \mu \mathrm{g} \mu \mathrm{l}^{-1}$ Cot-1 DNA (Roche, Mannheim, Germany); store at $\left(-20^{\circ} \mathrm{C}\right)$

- $3 \mathrm{M}$ sodium acetate solution; store at room temperature (RT; $19-26{ }^{\circ} \mathrm{C}$ )

- Absolute ethanol; use at $4{ }^{\circ} \mathrm{C}$

- NT enzyme: DNase-I/DNA polymerase-I (Invitrogen, Paisley, UK); store at

$-20{ }^{\circ} \mathrm{C}$ and thaw on ice immediately before use

- Electrophoresis loading dye (Fermentas, St. Leon-Rot, Germany)

- Ethanol: absolute 100, 90 and 70\%; use two times, then discard

- Formaldehyde solution: $1 \%$ formaldehyde in PBS (pH 7.2) containing

$50 \mathrm{mM} \mathrm{MgCl}_{2}$

- Acetone, for use in mild pre-treatment

- Nail varnish (clear)

- Rubber cement

- Wash solution (see REAGENT SETUP)

- Mounting medium (see REAGENT SETUP)

- FISH probes: generally store at $-20{ }^{\circ} \mathrm{C}$ unless otherwise stated

- RPMI-1640 (or equivalent) culture medium (Sigma): use 1:3 diluted in deionized water (optional)

- Giemsa stain (Merck, Darmstadt, Germany, cat. no. 1.09204.0500)

- Stock hypotonic solution (see REAGENT SETUP)

- Absolute methanol

- Glacial acetic acid

- $0.5 \times$ SSC, $2 \times$ SSC, $4 \times$ SSC etc. (see REAGENT SETUP)

$\cdot 10 \times$ NT buffer (see REAGENT SETUP)

- Hybridization buffer (see REAGENT SETUP)

$\cdot 10 \times$ dNTP mix (see REAGENT SETUP)

$\cdot 100 \times$ stock solution (see REAGENT SETUP)

- Pepsin stock solution (see REAGENT SETUP)

- Pepsin working solution (see REAGENT SETUP) $\triangle$ CRITICAL Chemical

reagents may be stored up to 4 weeks at $4{ }^{\circ} \mathrm{C}$, unless otherwise stated.

$\triangle$ CRITICAL Some materials are required only for G-banding and FISH;

therefore check in the procedure which materials you require.

\section{EQUIPMENT}

- Slides (see EQUIPMENT SETUP)

- 'Squeaky-clean' microscope slides (see EQUIPMENT SETUP)

- Research microscope (see EQUIPMENT SETUP)

- Routine microscope (see EQUIPMENT SETUP)

- Hybridization chamber (see EQUIPMENT SETUP)

- Hybridization bed (see EQUIPMENT SETUP)

- Laboratory oven for slide aging (G-banding) or drying (FISH)

-100-ml Coplin jars (glass) for staining and washing

- Plastic cover slips for probe detection (Qbiogene, Heidelberg, Germany)

- Cover slips: glass, grade $0,22 \times 60 \mathrm{~mm}$

REAGENT SETUP

$100 \times$ stock solution 1 part FUDR and 3 parts Urd $1 \mathrm{mg} \mathrm{ml}^{-1}$ for $100 \times$ stock solution. I CAUTION FUDR is a potential carcinogen and should be handled with care.

Thymidine Dissolve $50 \mathrm{mg}$ in $100 \mathrm{ml}$ for $100 \times$ stock solution. Filter-sterilize through $0.22-\mu \mathrm{m}$ filter. I CAUTION Thymidine is a potential carcinogen and should be handled with care.

Trypsin $0.5 \mathrm{~g} \mathrm{l}^{-1} /$ EDTA $0.2 \mathrm{~g} \mathrm{l}^{-1}$ (Invitrogen) for removal and dispersal of adherent cells. Store at $-20^{\circ} \mathrm{C}$. When it is used during simultaneous hypotonic treatment, dilute the latter accordingly.

Stock hypotonic solution $\mathrm{KCl} 5.59 \mathrm{~g} \mathrm{l}^{-1}(0.075 \mathrm{M})$ or Na citrate $9.0 \mathrm{~g} \mathrm{l}^{-1}$

$(0.9 \%)$. May be stored up to 1 year at $4{ }^{\circ} \mathrm{C}$. Working hypotonic solutions: mix $\mathrm{KCl}$ and $\mathrm{Na}$ citrate, e.g., 20:1, 10:1, 1:1, 1:10, 1:20 (vol/vol), shortly before use, allowing time to reach desired temperature. Optional variant hypotonic solution for use when all else fails: RPMI-1640 (or equivalent) culture medium: use 1:3 ( $\mathrm{vol} / \mathrm{vol})$ diluted in deionized water.

Fixative Absolute methanol and glacial acetic acid (3:1 vol/vol). Use fresh, but may be stored up to $4 \mathrm{~h}$ at $4{ }^{\circ} \mathrm{C}$. I CAUTION Glacial acetic acid is corrosive and must be handled using gloves inside a safety cabinet.

Trypsin stock solution $(\mathbf{7 0} \times)$ Dilute $10 \mathrm{ml}$ lyophilized 1:250 trypsin (Difco, Hamburg, Germany) to $17 \mathrm{ml}$ in PBS (pH 7.2). Store 500- $\mu$ l aliquots at $-20^{\circ} \mathrm{C}$. $\triangle$ CRITICAL Trypsin may be stored for up to approximately 6 months at $-20{ }^{\circ} \mathrm{C}$; thereafter, star activities predominate. Glass Coplin jars allow faster heat transfer than plastic, although the plastic jars are suitable for staining.

Giemsa stain Add 5-95 ml Sörensen A (1/15 $\left.\mathrm{M} \mathrm{KH}_{2} \mathrm{PO}_{4}\right)$ plus Sörensen B $\left(1 / 15 \mathrm{M} \mathrm{Na}_{2} \mathrm{PO}_{4}\right)$ buffer mixed $1: 1 \mathrm{vol} / \mathrm{vol}(\mathrm{pH} 6.85)$. Filter immediately before use $4 \times$ SSC NaCl $35.1 \mathrm{~g}$, Na citrate $17.7 \mathrm{~g}$ made up to 11 . Adjust to $\mathrm{pH} 7.2$. $0.5 \times$ SSC, $2 \times$ SSC, etc. Dilute from $4 \times$ SSC stock but monitor $\mathrm{pH}$. $10 \times \mathrm{dNTP}$ Mix $20 \mu \mathrm{l}$ from each of $20 \mathrm{mM}$ dATP, dCTP, dGTP and $6.6 \mu \mathrm{l}$ dTTP (Peqlab, Erlangen, Germany). Make up to $10 \mathrm{ml}$ with sterile deionized water. Store at $-20^{\circ} \mathrm{C}$.

10× NT buffer $1 \mathrm{ml} 1 \mathrm{M} \beta$-mercaptoethanol, $5 \mathrm{ml} 1 \mathrm{M}$ Tris- $\mathrm{HCl} \mathrm{pH} 7.8$, $200 \mu \mathrm{l} 2.5 \mathrm{M} \mathrm{MgCl}_{2}$ and $3.8 \mathrm{ml} \mathrm{H}_{2} \mathrm{O}$. Store aliquots at $-20{ }^{\circ} \mathrm{C}$.

Hybridization buffer Mix $5 \mathrm{ml}$ deionized formamide (GenomeLab sample


and $4 \mathrm{ml} 250 \mathrm{mM} \mathrm{Na}_{2} \mathrm{HPO}_{4}$ in $5 \times$ SSC. $\triangle$ CRITICAL In our experience, commercial hybridization buffers are not entirely reliable because they use impure formamides, which may cripple fluorescent probes.

Pepsin stock solution Dissolve $250 \mathrm{mg}$ pepsin (Sigma, cat. no. P7012) in $12.5 \mathrm{ml}$ deionized water. Freeze $500-\mu \mathrm{l}$ aliquots $\left(-20^{\circ} \mathrm{C}\right)$ and store for up to 6 months.

Pepsin working solution Dilute $500 \mu \mathrm{l}$ stock solution in $100 \mathrm{ml}$ deionized water containing $1 \mathrm{ml} 1 \mathrm{~N} \mathrm{HCl}$. 
Probe DNA Use $2 \mu \mathrm{g}$ for each $100-\mu l$ labeling reaction.

Wash solution $4 \times$ SSC with $0.1 \%$ Tween-20, molecular biology grade (Sigma). Slides may be popped into wash solution between any steps to prevent drying out.

Mounting medium Dissolve $50 \mathrm{ng} \mathrm{ml}^{-1} 4^{\prime}$,6-diamidino-2-phenylindole dihydrochloride (DAPI) in Vectashield antifade mounting medium (Alexis, Grünberg, Germany).

EQUIPMENT SETUP

Slides (with frosted ends for annotation) Wash the slides mechanically overnight using warm ion-free detergent, rinse two times in deionized water, oven-dry and leave overnight in ethanol (70\%). Slides should then be polished using a lint-free cloth (or non-shredding tissue) and stored wrapped in aluminum foil at $-20{ }^{\circ} \mathrm{C}$ until use.

'Squeaky-clean' microscope slides Commonly overlooked but essential is an adequate supply of 'squeaky-clean' microscope slides. ! CAUTION Commercially

\section{PROCEDURE} $400 \times$ ) if slow-growing (doubling time more than $72 \mathrm{~h}$ ).

? TROUBLESHOOTING

\section{(A) Suspension cultures} supernatants.

\section{(B) Adherent cultures} (treat as a biohazard). available 'pre-cleaned' slides are rarely clean enough, as even trace contaminants undermine chromosomal spreading or, even worse, if fluorescent, yield signals that intrude on FISH images.

Research microscope with the following brightfield objectives

(with numerical apertures as high as budgetary limitations permit): $10 \times, 63 \times$ (fluorescence), 63× (Planapochromatic). Ideally, a cytogenetics research microscope should be equipped with an automatic filter wheel and configured to an appropriate imaging system.

Routine microscope with phase-contrast (PC) illuminator and the following objectives: $\times 10(\mathrm{PC}), \times 40(\mathrm{PC})$ and $\times 50$ (brightfield-dry) for slide evaluation and preliminary analysis.

Hybridization chamber Sealed container with an internal shelf to separate slides (above) from humidifier, e.g., water-impregnated towels (below).

Hybridization bed Pre-warmed freezer block kept in incubator at $37^{\circ} \mathrm{C}$. Use during application of probes to slides.

\section{Harvesting and slide preparation $\bigcirc$ TIMING Harvesting $5 \mathrm{~h}$ (day 1 ) and slide making $1 \mathrm{~h}$ (day 2)}

1) Add colcemid to growing cultures for $1-4 \mathrm{~h}$ (normal growth) or overnight at quarter strength (i.e., dilute stock colcemid

$\triangle$ CRITICAL STEP Unlike routine primary cultures where culture conditions are standardized, cell lines often display highly individualized requirements and require regular feeding and density adjustment to maintain growth, with extreme densities often being inimical to growth. Remember: 'immortalized' does not mean the same as 'immortal'! The shock suffered by cells experiencing nutrient depletion, low $\mathrm{pH}$ values or extreme dilution may prove terminal. Although population doubling times normally range from 24 to $72 \mathrm{~h}$, exceptions at both ends are common. Mitotic metaphase, when chromosomes are clearly visible, lasts approximately $1 \mathrm{~h}$ or less, reducing the fraction of cells available for chromosome analysis. Metaphase enrichment is effected by exposing the growing cultures to the spindle poison colcemid for several hours (typically 1-4 h), or overnight in the case of slow-growing cells. Cultures are held in growth phase by feeding and, if necessary diluting/seeding out the day before harvest.

2| Harvest suspension cultures as described in option (A). Harvest adherent cultures as described in option (B).

(i) Aliquot evenly (e.g., $40 \mathrm{ml}$ fourfold into $10-\mathrm{ml}$ tubes) and centrifuge for $5 \mathrm{~min}$ at $400 \mathrm{~g}$ at RT. Discard the

(i) Agitate to dislodge mitoses and transfer the supernatants into centrifuge tubes (50 ml).

(ii) Meanwhile, rinse the remaining adherent cells with serum-free medium or PBS and discard the wash

(iii) Add just enough trypsin/EDTA to cover the cells and incubate briefly (5-15 min) at $37{ }^{\circ} \mathrm{C}$. Only when cells 'round up', shake vigorously (against a lab notebook etc., if need be) and decant after rinsing with supernatant from the centrifuge tube. Centrifuge the aliquots for $5 \mathrm{~min}$ at $400 \mathrm{~g}$ at RT. The serum present in the culture medium inactivates any remaining trypsin.

3| Perform hypotonic treatment. For cell lines listed in Supplementary Table 1, use the hypotonic treatment recommended therein. Note that the number of entries given under 'Times harvested' indicates the difficulty. For unlisted cell lines, perform initial 'ranging' hypotonic assessment guided by hypotonic treatments and times previously determined for similar types of cell lines, as given in Table 1.

$\triangle$ CRITICAL STEP The success of chromosome preparation depends on the choice of hypotonic treatment. Major variables are the composition of the hypotonic buffer, the duration of the treatment and temperature. However, variables often operate counterintuitively, calling for an empirical approach.

\section{? TROUBLESHOOTING}

4 Discard the supernatants into a biohazard container. Resuspend the cell pellets gently by flicking with your fingertip as vortexing may damage unfixed cells. Add $5 \mathrm{ml}$ working hypotonic solution to each tube for the desired duration and temperature.

5| Centrifuge at $400 \mathrm{~g}$ for $5 \mathrm{~min}$ at RT.

6| Discard the supernatants into a biohazard container. Resuspend the cell pellets as in Step 4. Gently -initially dropwise to prevent clumping-add $5 \mathrm{ml}$ ice-cold fixative to each tube while agitating the whole time to encourage mixing. 
PROTOCOL

TABLE 1 | Optimal hypotonic treatments for different tumors and species.

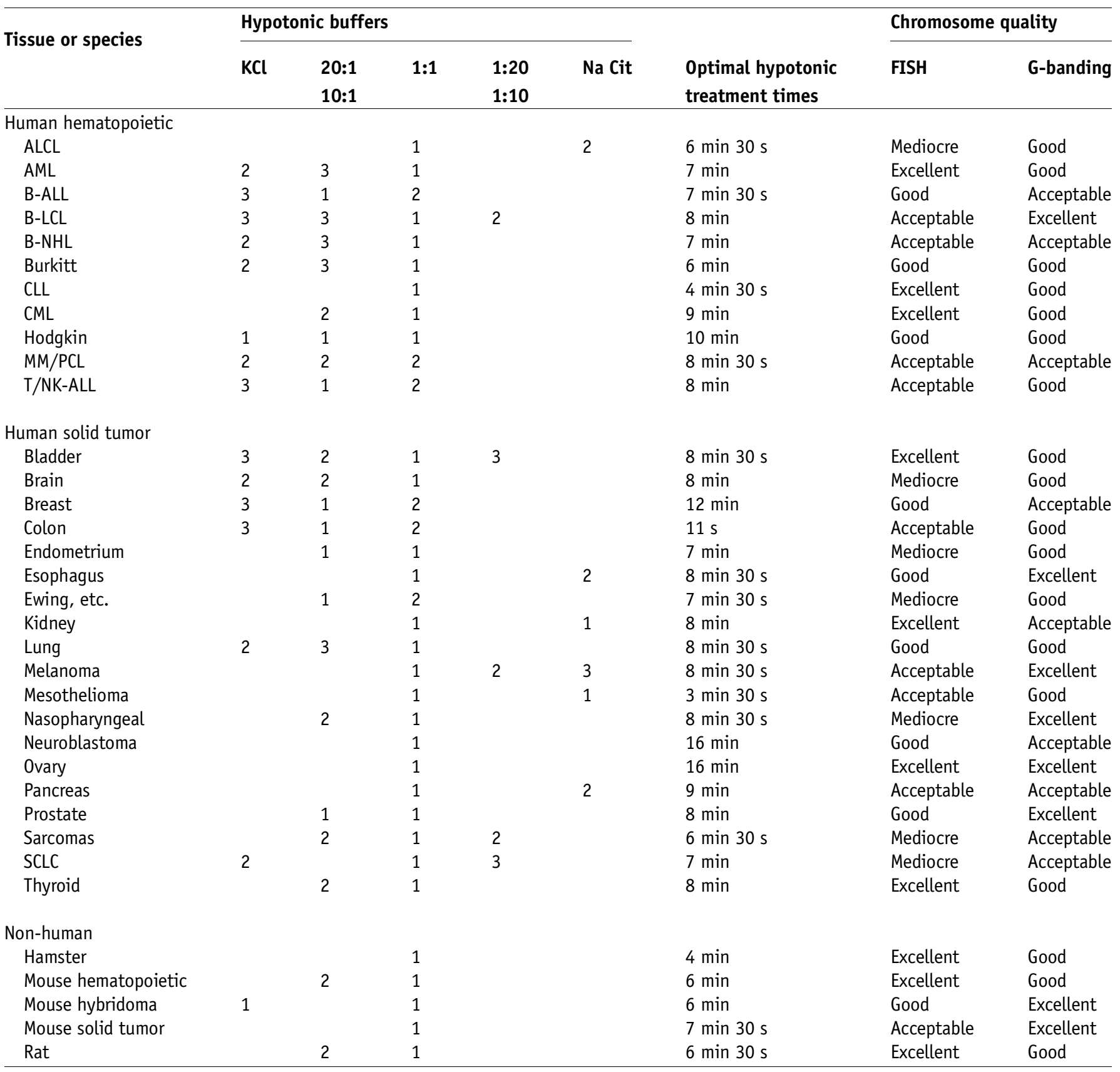

ALCL, anaplastic large cell lymphoma; AML, acute myeloid leukemia; B-ALL, B-acute lymphoblastic leukemia; B-LCL, B-lymphoblastoid cell-line; B-NHL, B-cell non-Hodgkin's lymphoma; CLL, chronic lymphocytic leukemia; CML, chronic myelogenous leukemia; MM/PCL, multiple myeloma/plasma cell leukemia; T/NK-ALL T-cell and natural killer cell leukemia/lymphoma. This table guides harvesting of cell lines not already listed in Supplementary Table 1. The hypotonic regimes listed here (numbered 1-3 according to proven efficacy) are intended as initial ranging treatments to be bracketed with milder and harsher conditions, respectively, to aid evidence-based assessment following that in Supplementary Table 2 . Hypotonic buffers comprise $0.075 \mathrm{M} \mathrm{KCl}$ or $0.9 \% \mathrm{Na}$ citrate, alone or mixed together, as indicated; optimal hypotonic treatment times are means rounded to the nearest $30 \mathrm{~s}$; chromosome quality assessments are given separately for FISH and G-banding, as these end-points are occasionally discrepant.

7| Store on ice for approximately $1 \mathrm{~h}$ to allow thorough fixation.

8| Remove from ice and place in a stand for $10 \mathrm{~min}$ to allow tubes to approach RT (to minimize clumping), then centrifuge for 5 min at $400 \mathrm{~g}$ at RT. Resuspend in fresh fixative.

9| Tubes should be stored overnight at $4{ }^{\circ} \mathrm{C}$ in a refrigerator or cold room.

10| The next day, allow tubes to reach RT.

11| Centrifuge at $400 \mathrm{~g}$ for 5 min at RT. Repeat a further two times, discarding each supernatant. 
PROTOCOL

TABLE 2 | RAMOS (DSM ACC 603).

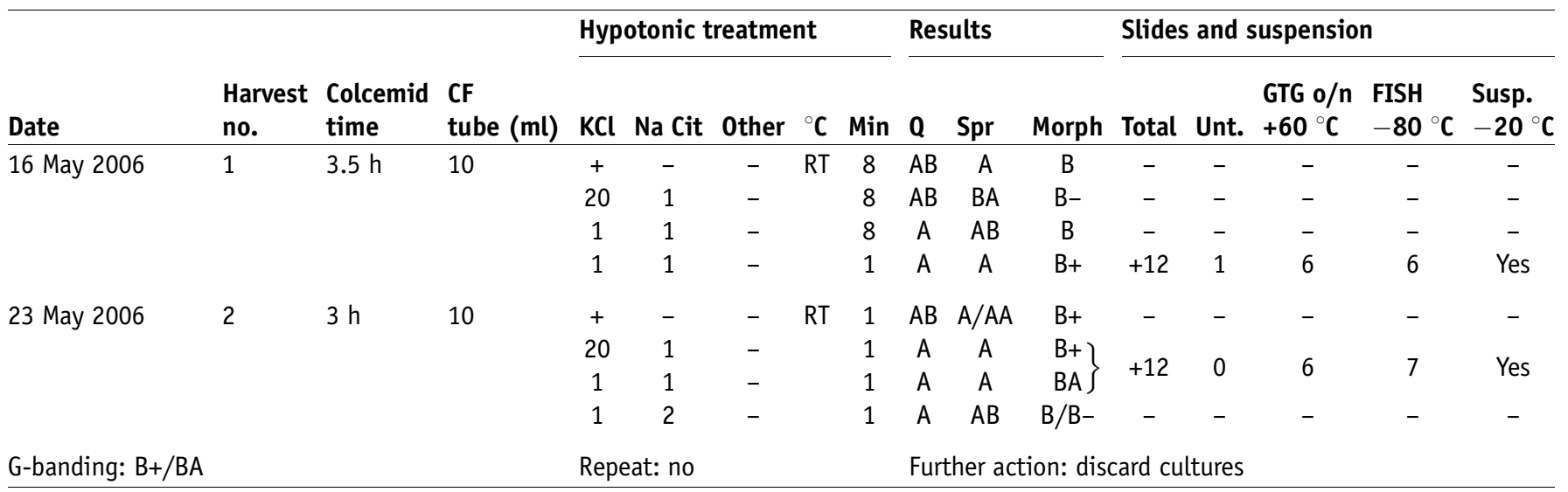

Abbreviations: CF, centrifuge; FISH fluorescence in situ hybridization; GTG, G-banding with trypsin-Giemsa; Morph(ology); 0/N, overnight; Q(uality); RT, room temperature, Spr(eading); Susp(ension of fixed cells); Unt(reated).

12| Resuspend finally in approximately $1 \mathrm{ml}$ fixative, adjusting the actual volume until slightly cloudy.

13| Place chilled $\left(-20^{\circ} \mathrm{C}\right)$ pre-cleaned slides (one per harvest tube) onto a freezer-block sloped gently away from operator by placing it on top of a single pipette or something similar.

14| Moisten slides by heavy breathing (humidity encourages chromosome spreading).

? TROUBLESHOOTING

15 Place two drops of suspension symmetrically onto each slide from approximately $30 \mathrm{~cm}$ above, the first drop just below the frosted zone, the second further along. Do not flood.

16| Breathe on the slide again (optional to encourage spreading).

17| To improve spreading further, you can gently flame at the edges using a camping stove or Bunsen burner. This is optional and is intended merely to encourage ignition rather than baking dry.

18| Label the slides and air-dry for approximately 15 min; allow slides to stand near-vertically until dry.

PAUSE POINT Slides can be stored dry for several hours before assessment.

19| Examine slides using PC microscopy and assess each hypotonic treatment individually. Record assessments in a table similar to Supplementary Table 2. Table 2 gives an example of the type of assessment that can be made.

$\triangle$ CRITICAL STEP Harvesting is a trial-and-error process. Optimization of harvest conditions depends on accurate evidence-based assessment of results. Thus, keep note of (i) frequency of metaphases relative to other nuclei-mitotic scarcity may indicate an overly harsh hypotonic regime as well as insufficient mitotic arrest or proliferative deficit; (ii) spreading, which should render each chromosome visible without 'cross-overs' or undue breakage - the last also caused by overlong/overly harsh hypotonic regimes; and (iii) morphology, to which chromosomal length, parallel arms and 'unfuzziness' without refractility under PC illumination also contribute.

? TROUBLESHOOTING

20| If results are satisfactory, mix suspensions from successful harvests and adjust volumes if necessary after centrifugation.

21| Prepare slides and label them clearly.

\section{Analysis}

22| Apportion slides for simple staining and immediate FISH (no extra treatment), G-banding (bake overnight at $60{ }^{\circ} \mathrm{C}$, see option (A) below) and future FISH (store at $-80^{\circ} \mathrm{C}$, see options (B) and (C) below). Option (B) describes probe labeling. Option (C) describes FISH. When making your own probes, perform (B), then (C). If you are using commercial or pre-prepared probes, perform option (C) only.

- PAUSE POINT Any remaining suspension can be stored at $-20{ }^{\circ} \mathrm{C}$ for up to approximately 5 years.

\section{(A) Trypsin G-banding TIMING 1 h}

(i) Fresh slides are too labile for immediate G-banding and require aging by heating overnight at $60{ }^{\circ} \mathrm{C}$ (or in extremis for 1-2 $\mathrm{h}$ at $\left.90{ }^{\circ} \mathrm{C}\right)$. Hence, prepare to treat $6-8$ slides containing an adequate supply of well-spread metaphases. 
(ii) Prepare three glass Coplin jars containing $500 \mu$ trypsin in $70 \mathrm{~mL}$ PBS (pH 7.2), ice-cold PBS (pH 6.8) to stop enzymatic activity and 5\% Giemsa in PBS (pH 6.8) at RT, respectively.

(iii) Pre-heat Coplin jar containing trypsin/PBS in the water bath at $37^{\circ} \mathrm{C}$ until the internal temperature has equilibrated. Check using a validated thermometer.

$\Delta$ CRITICAL STEP Trypsin digestion is highly temperature dependent. It is, therefore, vital to check that the temperature remains constant at $37^{\circ} \mathrm{C}$.

(iv) Estimate optimal trypsin time by dipping the first slide halfway into the first Coplin jar initially for $10 \mathrm{~s}$, followed by the whole slide for the remaining $10 \mathrm{~s}$.

(v) Quench trypsin activity by plunging the slide into the second Coplin jar, containing ice-cold PBS, for a few seconds.

(vi) Shake the slide to remove PBS droplets. Stain in the Coplin jar containing Giemsa solution for 15 min at RT.

(vii) Shake the slide and rinse briefly in deionized water. Blot dry using recycled paper towels. Leave for a further $10 \mathrm{~min}$ to air dry.

(viii) Estimate optimal trypsin time by microscopically scanning the slide for quality metaphases at low power (approximately $\times 100$ total magnification) under bright-field settings. Closely examine those selected at higher power $(\times 500)$ using an Epiplan or equivalent high-resolution dry objective. Decide whether the optimal trypsin time lies within the 10-20 s range spanned by the test slide. If yes, repeat Steps (i)-(vii). If unsatisfactory, repeat Steps (i)-(vii) using longer (e.g., 30/45 s) or shorter (e.g., 3/6 s) trypsin test times, as appropriate until the optimal time is determined.

? TROUBLESHOOTING

(B) Non-isotopic FISH labeling by NT $\bigcirc$ TIMING Duration $7 \mathbf{h}$

(i) Mix the following on ice: $2 \mu \mathrm{g}$ probe DNA, $10 \mu \mathrm{ldNTP}$ mix, $10 \mu$ l hybridization buffer $(10 \times)$ and sterile deionized water to make up to $90 \mu \mathrm{l}$.

(ii) Add $10 \mu \mathrm{L}$ NT enzyme kept at $4{ }^{\circ} \mathrm{C}$.

(iii) Incubate for $120 \mathrm{~min}$ at $15^{\circ} \mathrm{C}$.

(iv) Remove a 3- $\mu$ l aliquot and put the remainder on ice.

(v) Mix the aliquot together with $5 \mu$ l loading dye after denaturing for 5 min at $95{ }^{\circ}$. Briefly collect by centrifugation in a microfuge for a couple of seconds at maximum speed (approximately 12,000 g) and electrophorese in a $1.5 \%$ agarose gel containing ethidium bromide for 30 min at $80 \mathrm{~V}$.

I CAUTION Ethidium bromide is potentially mutagenic and carcinogenic. Dispose of gel in a biohazard container.

(vi) If the bandsmear is satisfactory (100-500 bp), proceed with Step (vii). If the bandsmear is longer than $500 \mathrm{bp}$, incubate for another $60 \mathrm{~min}$. If the bandsmear remains too long, add fresh NT enzyme or just DNA polymerase-I alone. ? TROUBLESHOOTING

(vii) Add $10 \times$ stop buffer.

(viii) Precipitate labeled DNA by adding 5-10 $\mu \mathrm{l}$ human Cot-1 DNA (in particular for genomic large-insert probes), $10 \mu \mathrm{l}$ herring sperm DNA, $12 \mu \mathrm{l} 3 \mathrm{M}$ sodium acetate and $250 \mu \mathrm{l}$ ice-cold absolute ethanol to the reaction mix.

(ix) Precipitate at $-80^{\circ} \mathrm{C}$ for 30 min.

(x) Spin down (hinges pointing inward to enable subsequent pellet identification) in a microfuge at maximum speed (approximately $12,000 \mathrm{~g}$ ) for $30 \mathrm{~min}$ at $4^{\circ} \mathrm{C}$.

(xi) Visualize the pellet. Gently remove and discard the supernatant.

(xii) Gently add $200 \mu \mathrm{l} 70 \%$ ethanol. Centrifuge again for 5 min at $12,000 \mathrm{~g}$ at $4{ }^{\circ} \mathrm{C}$.

(xiii) Dry the pellet using a vacuum centrifuge.

(xiv) Resuspend in $50 \mu \mathrm{l}$ sterile deionized water by thorough vortexing and store or proceed with FISH (option (C)). - PAUSE POINT Can be stored at $-20{ }^{\circ} \mathrm{C}$ until required.

(C) FISH $\bigcirc$ TIMING Dehydration (days 1-2) approximately $17 \mathrm{~h}$ overnight; probe preparation/application 3-6 $\mathrm{h}$ for 1-10 slides (day 2); stringency wash and slide preparation 1-1.5 $\mathrm{h}$ for 1-10 slides (day 3)

(i) If possible, use fresh slides ( $1-7 \mathrm{~d}$ old) or slides cryostored for up to 2 years at $-80{ }^{\circ} \mathrm{C}$. Although frozen slides may be usable after 10 years, results are unreliable.

(ii) To reduce the background signal you can pre-incubate slides for 2 min in acidified pepsin solution at $37{ }^{\circ} \mathrm{C}$, rinse in PBS (pH 7.2) for 3 min at RT and post-fix slides, held flat, in 1\% formaldehyde solution for 10 min at RT using plastic cover slips. Rinse in PBS (pH 7.2) for 3 min at RT before proceeding. This step is optional.

(iii) Dehydrate slides by moving them through an alcohol series of $2 \mathrm{~min}$ in $70 \%(\times 2), 90 \%(\times 2)$ and $100 \%$ ethanol in Coplin jars.

(iv) Allow slides to dry overnight in an oven set at $42{ }^{\circ} \mathrm{C}$.

(v) De-proteinize slides in acetone for $10 \mathrm{~min}$. 
(vi) Prepare to denature the slides by heating a Coplin jar containing $30 \mathrm{ml} 2 \times \mathrm{SSC}$ plus $70 \mathrm{ml}$ formamide to $72{ }^{\circ} \mathrm{C}$. Check the internal temperature using a validated thermometer. Denature the slides for $2 \mathrm{~min}$ at $72{ }^{\circ} \mathrm{C}$. Quench in pre-chilled $\left(-20^{\circ} \mathrm{C}\right) 70 \%$ ethanol for 2 min.

$\triangle$ CRITICAL STEP The denaturation temperature is critical. Avoid mass denaturation: each additional slide reduces the temperature within the Coplin jar by approximately $1{ }^{\circ} \mathrm{C}$. If high throughput is desired, slides should be pre-warmed.

(vii) Repeat the alcohol series in Step (iii).

(viii) Renew slide labels and varnish (to prevent subsequent eradication).

(ix) Place slides on a block pre-warmed to $37^{\circ} \mathrm{C}$, or on a thermostatically controlled hotplate.

(x) Remove FISH probe(s) from the freezer or refrigerator.

(xi) For commercial probes, consult the manufacturer's own protocols and adapt what follows accordingly. For home-made probes, take probes made as described in option (B) and denature the probe aliquots. Pipette the desired volume (approximately 7-10 $\mu \mathrm{l}$ ) of probe into a microfuge tube (sterile) and denature for $5 \mathrm{~min}$ at $72{ }^{\circ} \mathrm{C}$ in a water bath. (Note that if recommended by the manufacturer, omit probe denaturation.) The concentration of probe DNA depends on its target size: whole-chromosome (painting) probes or labeled BAC clones may be used at correspondingly higher concentrations than shorter fosmid/cosmid and plasmid probes. Probes including non-coding sequences (e.g., BAC clones) should also contain Cot-1 DNA.

(xii) Collect the probe by brief centrifugation in a microfuge (approximately $12,000 \mathrm{~g}$ for a couple of seconds) and then pre-hybridize for $15-60 \mathrm{~min}$ at $37^{\circ} \mathrm{C}$ in a second water bath. This incubation permits 'noisy' probe repeating DNA sequences to 'hybridize out' with matching sequences in Cot-1 DNA.

(xiii) Apply the probe using shortened micropipette tips (sterile) to drop 8-12 $\mu$ l probe carefully (making up the volume with Hybrizol, if necessary) onto each half of the slide. Two hybridizations may thus be performed on each slide (separated by a drop of Hybrizol, to inhibit mixing). Cover slides carefully with glass cover slips, tapping out any bubbles, and seal with rubber cement.

(xiv) Hybridize by placing slides carefully in a moistened and sealed hybridization chamber. Leave overnight (or for up to $72 \mathrm{~h}$ ) in an incubator (preferably humidified) at $37^{\circ} \mathrm{C}$.

(xv) After hybridization carefully remove the rubber cement and cover slips in $2 \times$ SSC using tweezers.

(xvi) Perform stringency washing by washing the slides for 5 min at $72{ }^{\circ} \mathrm{C}$ in $0.5 \times \mathrm{SSC}$. ? TROUBLESHOOTING

(xvii) If using digoxigenin labeled probes, briefly pre-wash in wash solution at RT, and then shake excess wash free. $\triangle$ CRITICAL STEP Do not allow slides to dry out until dehydration.

(xviii) To each slide, apply $40 \mu \mathrm{l}$ anti-digoxigenin antibody haptenized with fluorescein-isothiocyanate (FITC) (Qbiogene, Heidelberg, Germany) and cover with a plastic cover slip. Incubate for $15-30$ min at $37{ }^{\circ} \mathrm{C}$ in hybridization chamber.

(xix) Wash for $5 \min (\times 3)$ in wash solution at RT in subdued light.

(xx) Perform dehydration via an alcohol series, as described in Step (iii) above, but in subdued light.

(xxi) Mount and seal using shortened micropipette tips. Carefully place three drops DAPI/Vectashield mount, each of approximately $20 \mu \mathrm{l}$, along the slide. Apply cover slip and tap out any large bubbles using the blunt end of a pencil or something similar. Seal with nail varnish. Allow varnish to dry.

(xxii) Visualize slides at high power under oil immersion with $a \times 63$ objective with a high numerical aperture under epifluorescence.

? TROUBLESHOOTING

\section{? TROUBLESHOOTING}

If quantities and/or chromosome spreading is unsatisfactory, consult Table 1, which lists the success rates of a variety of hypotonic treatments for different types of cell line. Although 1:1 KCl:Na citrate offers the best chance of success, a significant minority of cell lines produce the best results using other treatments. Optimal mean hypotonic times range from 4 to 16 min. If chromosome morphology doggedly remains poor, treat cultures overnight with FUDR/Urd. Next morning, resuspend in fresh medium with added thymidine to reverse the blockade and harvest 7-9 $\mathrm{h}$ later.

\section{FISH}

The twin problems commonly encountered when using FISH probes (commercial or tailor-made) are weak signal intensity and/or high background owing to weak probe labeling, insufficient DNase-I digestion, wash stringency or chromosome denaturation. To test for these alternatives, repeat the stringency wash in Step 22C (xvi) but with either $2 \times$ or $1 \times$ SSC in the wash buffer. In parallel, repeat the slide denaturation, increasing the denaturation time to $4 \mathrm{~min}$. If neither alteration brings any improvement and the probe is new and untested not old and infrequently used, it is likely that the probe is weak. With advanced imaging systems incorporating a camera of high sensitivity, it is often possible to capture images from probe signals invisible to the naked eye. In the case of new commercial probes, the supplier should be contacted. On the other hand, FISH experiments are sometimes plagued by high background signal, or noise. Commercial probes are usually relatively free from this problem. 
Increasing the wash stringency by reducing the SSC concentration to $0.1 \times$ may help. Alternatively, adding Cot-1 DNA to the hybridization mix may help to reduce hybridization noise. With non-commercial probes, excessive noise may often be cured by reducing the probe concentration. Normal DNA concentrations for single-locus probes should range from 2-6 to 10-20 ng $\mu^{-1}$ for painting probes. Non-specific noise caused by either autofluorescence or protein-protein binding after antibody staining may be reduced by additional slide pre-treatment by incubation in pepsin solution.

\section{? TROUBLESHOOTING}

Troubleshooting advice can be found in Table 3 .

TABLE 3 | Troubleshooting table.

\begin{tabular}{lll}
\hline Step & Problems & Causes \\
\hline 1 & Insufficient metaphases & Long cell population doubling time
\end{tabular}

Depletion owing to overly harsh
hypotonic regime

Improve cell culture conditions: feed or supplement with growth factors

\section{hypotonic regime}

3

Insufficient spreading

14

19

Poor morphology

Disappointing G-banding

FISH chromosomes 'puffy' cytogenetic data

Insufficient humidity

Mycoplasma contamination: eliminate using suitable antibiotics (ref. 18).

Reduce hypotonic times

Perform in microfuge tubes to reduce volumes and centrifuge times

Use milder (less dilute) hypotonics

Perform at $4^{\circ} \mathrm{C}$ on ice

Hypotonic regime too mild

Increase hypotonic times to $15-30 \mathrm{~min}$

Perform in microfuge tubes to reduce volumes and centrifuge times

Use harsher (more dilute) hypotonics, e.g., $0.66 \mathrm{M} \mathrm{KCl} / 0.8 \%$ Na citrate

Perform at $37{ }^{\circ} \mathrm{C}$ in water bath

Breathe on slides before and after dropping

Ensure slides are pre-chilled to $-20{ }^{\circ} \mathrm{C}$

Increase humidity by dropping onto slides held above a hot (hotter than $70^{\circ} \mathrm{C}$ ) water bath

Flame slides

Excessive surface tension on slide

\section{Sub-optimal chromosome} condensation

Metaphases too young or fragile Slides too old or deteriorated

\section{Trypsin expired}

Slides too young

Slides too old

Probe labeling

Competitor DNA

Post-hybridization wash

Cell line contains subclones

Cell line cross-contaminated or misidentified
Ensure slides are 'squeaky clean': pre-clean in $1 \% \mathrm{HCl}$, rinse in $\mathrm{H}_{2} \mathrm{O}(\times 2)$ followed by ethanol wash, and polish using lint-free cloth

Try a different hypotonic: minimize Na citrate content

Treat cultures overnight with FUDR/Urd. Next morning, resuspend in fresh medium with added thymidine to reverse the blockade and harvest 7-9 $\mathrm{h}$ later

Increase aging by longer pre-baking times up to $24 \mathrm{~h}$ (G-banding)

Use fresh slides

Improve slide storage: store at $-80^{\circ} \mathrm{C}$, ideally airtight to combat icing

Store suspensions in ethanol: resuspend in fix just before slide-making

Trypsin lasts approximately 6 months at $-20^{\circ} \mathrm{C}$ : prepare fresh aliquots

Reduce slide denaturation times (FISH)

Age overnight

Drop fresh slides from suspensions

See above ? TROUBLESH00TING section on FISH

Clone by limiting dilution: test first whether cells tolerate low densities

Discard all but earliest stocks: retest these, if false, discard too 


\section{ANTICIPATED RESULTS}

Cancer cytogenetics distinguishes four major types of rearrangement in tumor cells. (i) Primary changes are often chromosome translocations, e.g., $\mathrm{t}(9 ; 22)(\mathrm{q} 34 ; \mathrm{q11})$ in $\mathrm{CML}$, where fusion of breakpoint cluster region (BCR) (at chromosome 22q11) with Abelson murine leukemia viral oncogene (ABL) (at chromosome 9q34) and occurs in almost every patient at early disease stages. (ii) Secondary changes are usually numerical changes affecting whole chromosomes or regions thereof, e.g., i(17)(q10) increasing copy number of the chromosome $17 q$ (long arm) region, which accompanies $t(9 ; 22)$ at advanced disease stages. (iii) Apparently random changes which may be structural or numerical. Although it is sometimes believed that random changes mark genetic instability during disease progression or in vitro culture, no longitudinal studies using cloned material (to exclude outgrowth of cryptic diverse subclones) have been published to verify this. Indeed, both longitudinal data ${ }^{15}$ and comparison of subclones carrying complex rearrangements ${ }^{16}$ indicate extreme stability in culture. (iv) Chromosome changes are tentatively associated with adaptation to growth in culture, although this phenomenon remains poorly documented.

Table 2 summarizes the results of harvesting the RAMOS Burkitt lymphoma cell line for which G-banding and FISH are depicted in Figure 4, yielding typical results for a hematopoietic malignant cell line. The first harvest indicated superiorities of short (1-min) harvest times and 1:1 KCl:Na citrate hypotonic buffer. Using these as guides, the second harvest was performed throughout using 1-min harvest times, supplementing the standard buffers with 1:2 KCl:Na citrate. However, the best results were again obtained with 1:1, supplemented with 20:1. These suspensions were pooled and used to produce slides for G-banding and FISH that are illustrated in Figure 4, which depicts how analysis of $\mathrm{t}(8 ; 14)(\mathrm{q} 24 ; \mathrm{q} 32)$ in the RAMOS Burkitt lymphoma cell line confirms that it involves rearrangement of both MYC and IGH, as is typical for this entity.

Note: Supplementary information is available via the HTML version of this article.

COMPETING INTERESTS STATEMENT The authors declare that they have no competing financial interests.

Published online at http://www.natureprotocols.com

Reprints and permissions information is available online at http://npg.nature.com/ reprintsandpermissions

1. Drexler, H.G. The Leukemia-Lymphoma Cell Line Facts Book (Academic Press, London, 2001).

2. Drexler, H.G. Guide to Leukemia-Lymphoma Cell Lines (DSMZ Braunschweig, Germany, 2005).

3. MacLeod, R.A. et al. Widespread intraspecies cross-contamination of human tumor cell lines arising at source. Int. J. Cancer 83, 555-563 (1999).

4. Mrózek, K., Heinonen, K. \& Bloomfield, C.D. Clinical importance of cytogenetics in acute myeloid leukaemia. Baillieres Best Pract. Res. Clin. Haematol. 14, 19-47 (2001).

5. Swansbury, J. Acute lymphoblastic leukemia: background. Methods Mol. Biol. 220, 59-71 (2003).

6. Muyrers-Chen, I. et al. Expression of leukemic MLL fusion proteins in Drosophila affects cell cycle control and chromosome morphology. Oncogene 23, 8639-8648 (2004).

7. Caspersson, T., Zech, L. \& Johansson, C. Differential binding of alkylating fluorochromes in human chromosomes. Exp. Cell Res. 60, 315-319 (1970).

8. Seabright, M. Improvement of trypsin method for banding chromosomes. Lancet 1, 1249-1250 (1973).

9. Rowley, J.D. Letter: a new consistent chromosomal abnormality in chronic myelogenous leukaemia identified by quinacrine fluorescence and Giemsa staining. Nature 243, 290-293 (1973).
10. Cremer, T., Lichter, P., Borden, J., Ward, D.C. \& Manuelidis, L. Detection of chromosome aberrations in metaphase and interphase tumor cells by in situ hybridization using chromosome-specific library probes. Hum. Genet. 80, 235-246 (1988).

11. Lichter, P., Cremer, T., Borden, J., Manuelidis, L. \& Ward, D.C. Delineation of individual human chromosomes in metaphase and interphase cells by in situ suppression hybridization using recombinant DNA libraries. Hum. Genet. 80, 224-234 (1988).

12. Lichter, P. Multicolor FISHing: what's the catch? Trends Genet. 12, 475-479 (1997).

13. ISCN. An international system for human cytogenetic nomenclature: report of the Standing Committee on Human Cytogenetic Nomenclature. Birth Defects Orig. Artic. Ser. 21, 1-117 (1985).

14. Drexler, H.G., Dirks, W.G., Matsuo, Y. \& MacLeod, R.A. False leukemia-lymphoma cell lines: an update on over 500 cell lines. Leukemia 17, 416-426 (2003).

15. Tosi, S. et al. Characterization of the human myeloid leukemia-derived cell line GF-D8 by multiplex fluorescence in situ hybridization, subtelomeric probes, and comparative genomic hybridization. Genes Chromosomes Cancer 24, 213-221 (1999).

16. MacLeod, R.A. et al. Karyotypic dissection of Hodgkin's disease cell lines reveals ectopic subtelomeres and ribosomal DNA at sites of multiple jumping translocations and genomic amplification. Leukemia 14, 1803-1814 (2000).

17. Poulsen, T.S. et al. Detection of illegitimate rearrangement within the immunoglobulin locus on 14q32.3 in B-cell malignancies using end-sequenced probes. Genes Chromosomes Cancer 32, 265-274 (2001).

18. Uphoff, C.C. \& Drexler, H.G. Eradication of mycoplasma contaminations. Methods Mol. Biol. 290, 25-34 (2005). 